\title{
Protée
}

\section{D'un art du mouvement à un art en mouvement : du cinéma au théâtre de l'image}

\section{Chantal Hébert et Irène Perelli-Contos}

Volume 28, numéro 3, 2000

Mélancolie entre les arts

URI : https://id.erudit.org/iderudit/030605ar

DOI : https://doi.org/10.7202/030605ar

Aller au sommaire du numéro

\section{Éditeur(s)}

Département des arts et lettres - Université du Québec à Chicoutimi

ISSN

0300-3523 (imprimé)

1708-2307 (numérique)

Découvrir la revue

Citer cet article

Hébert, C. \& Perelli-Contos, I. (2000). D’un art du mouvement à un art en mouvement : du cinéma au théâtre de l'image. Protée, 28(3), 65-74.

https://doi.org/10.7202/030605ar
Résumé de l'article

Dans cet article nous proposons d'éclairer certaines modalités du théâtre de l'image, pour tenter de saisir comment ce théâtre, créateur d'images multidimensionnelles, est en train de bouleverser le régime habituel de vision du spectateur et son rapport traditionnel à la représentation. Pour ce faire, nous puisons nos exemples dans Vinci, oeuvre emblématique de celui qui, au Québec, a la réputation de véritable " faiseur d'images ", l'auteur, metteur en scène, cinéaste et comédien Robert Lepage. Ce choix est motivé par le fait que l'oeuvre de cet artiste prolifique et polyvalent est non seulement représentative de la nouvelle poétique qui semble sourdre de l'ère informatique et de la culture médiatique, mais constitue un terrain de choix permettant de mieux rendre compte des conséquences épistémologiques du croisement des médias dans la production culturelle contemporaine et de la manière dont le théâtre de l'image participe au renouveau de l'écriture et de la pensée. 


\section{D'UN ART DU MOUVEMENT À UN ART EN MOUVEM ENT: DU CINÉMA AU THÉÂTRE DE L'IMAGE \\ ChANTAL H ÉBERT ET IRÈn E PERELLI-CONTOS}

Penser le théâtre dans une perspective médiatique ou intermédiatique, c'est chercher à le rapprocher - et à le confronter - de ce à quoi il s'oppose d'ordinaire, soit des pratiques qui, comme le cinéma et la vidéo par exemple, reposent sur une infrastructure technologique. À l'aube du XXIe siècle pourtant, le théâtre s'est à ce point modifié sous la pénétration technologique qu'il n'est désormais plus possible de l'approcher comme un "art pur», si tant est qu'il ait déjà été possible de le faire. Les processus d'échange, de métissage, d'hybridation sont si fréquents et si diversifiés qu'on doit bien tenir compte du réseau d'influences et d'interférences qui a fini par se tisser et par introduire sur la scène non seulement de nouvelles techniques, mais surtout - et c'est ce qui nous intéresse ici - de nouvelles modalités de représentation. Car si, à travers l'histoire, la scène a toujours tiré profit des progrès techniques - ce qui se comprend en raison du statut éminemment matériel de celle-ci -, on ne s'étonnera pas qu'elle ait été perméable à l'évolution incessante des technologies et on comprendra que ce qui aura le plus profondément marqué le théâtre des vingt dernières années - ces années dites postmodernes - ce sera sans doute l'interaction effective des pratiques et des techniques médiatiques qui ont infiltré la pratique scénique. Mais au progrès technique correspond aussi l'intégration ou l'absorption des «valeurs» de la technologie. Or avec les nouvelles techniques d'imagerie, envisagées comme nouvel outil d'écriture, un ordre visuel inhabituel a fait son apparition dont est tributaire l'écriture scénique.

Ainsi donc à l'heure de la mondialisation des médias, de l'expansion rapide de nouvelles technologies, de l'éclatement des frontières entre les cultures et les disciplines artistiques, le théâtre au Québec, aussi bien qu'en Europe ou qu'aux États-Unis, s'est mis à l'exploration de formes nouvelles et de contenus nouveaux, s'essayant «à représenter le monde avec des règles qui, comme l'écrit Jean-Pierre Ryngaert, ne dérivent pas toutes d'Aristote " 1 . Or parmi les différentes tendances ou explorations tentées au cours des vingt dernières années ${ }^{2}$, force est de reconnaître la place et l'importance de l'une d'entre elles, qui a contribué à redéfinir la théâtralité, par la création d'un langage théâtral différent, imagé et 
polysémique: le théâtre de l'image. Accordant la primauté à l'image, comme son nom l'indique, et à ses composantes (lumières, objets, formes, mouvements, rythmes, etc.), l'originalité de ce théâtre tient dans son écriture scénique qui s'offre comme un jeu combinatoire d'objets, de techniques, de modèles hétéroclites, autrement dit d'éléments à assembler; éléments empruntés à la fois au réel, aux différentes disciplines, aux autres arts, aux autres cultures, aux technologies actuelles, etc. L'exercice d'assemblage peut s'apparenter à un puzzle transformable dont plusieurs pièces, toutefois, sont manquantes et dont même les règles d'assemblage restent parfois à découvrir. On se trouve ainsi devant une nouvelle manière d'écrire directement sur la scène avec d'autres éléments que les mots. Cette écriture, qualifiée de «magique» par certains, au lieu de redoubler le monde, donne à voir des images aux structures spatiales inédites, aux textures étranges, aux perspectives éclatées s'ouvrant sur des imaginaires singuliers dont les logiques et les topologies nous sont encore peu familières. Il en résulte une crise de la représentation susceptible d'être interprétée comme une blessure à caractère mélancolique et qui mérite, croyons-nous, de retenir l'attention.

Or nous savons que cette crise remonte au moins au début du siècle et qu'elle a été enclenchée, entre autres, par l'avènement du cinéma. On n'a pour s'en convaincre qu'à se rappeler les expériences des avantgardes russe et italienne, pensons à Meyerhold et à Marinetti. En 1928, par exemple, Meyerhold était préoccupé de «cinéfier la scène: [de] la rendre si mobile qu'elle permette de donner une succession rapide de tableaux» ${ }^{3}$. "Les étonnantes possibilités dont dispose le cinéma, disait-il, (transporter l'action d'un pays à un autre, sauter du jour à la nuit, etc.) le théâtre peut, lui aussi en user commodément" ${ }^{4}$, et ce dans le but de "créer un théâtre et une scène dont le dynamisme et la capacité d'impact seront sans précédent " ${ }^{5}$. De même, le rêve des futuristes n'était-il pas «d'atteindre au théâtre [...] la rapidité et la force de stupéfaction du cinéma " 6 , tout en étant «indépendant du monde phénoménal» ${ }^{7}$. Ce postulat esthétique d'un «art pur, délivré des scories de la logique narrative et des lois de l'orthodoxie formelle» ${ }^{8}$, a nourri les recherches des futuristes, ouvrant vraisemblablement la voie au théâtre de l'image actuel. Bref, tantôt inspiré par le cinéma, tantôt par les arts visuels ou encore par le music-hall, le théâtre s'est transformé, faisant entrer en scène le spectateur qui était jusque-là, en raison du "quatrième mur", tenu à bonne distance des images du monde. Depuis et surtout avec les nouvelles technologies, il a le pouvoir de faire pénétrer - de faire plonger - créateurs et spectateurs dans des univers insoupçonnés, ceux, dirait Paul Valéry, des phénomènes de l'imagerie mentale. Il a, de ce fait, le pouvoir de remettre définitivement en cause l'espace traditionnel de représentation et, avec lui, nos modes de perception et de (re)connaissance.

Nous proposons donc ici d'éclairer certaines modalités du théâtre de l'image, pour tenter de saisir comment ce théâtre, créateur d'images multidimensionnelles, est en train de bouleverser le régime de vision du spectateur et, par conséquent, son rapport au monde. Pour ce faire, il nous faut passer à l'étude de l'activité de quelques-unes au moins de ces images, qui ont délaissé les structures linéaires et réalistes de la représentation au profit d'associations inattendues qui se répondent ou s'opposent dans une structure en réaménagement permanent, permettant ainsi à la représentation de se poser pas plus comme représentation concrète, réaliste et achevée du monde sensible que comme sa recomposition originale et, de ce fait, de nous apprendre à voir autrement ce monde qui nous entoure. À l'issue de cette constatation qui résume en quelque sorte ce qui semble être l'aboutissement des révolutions scéniques du XX siècle, on peut se demander si on ne serait pas bel et bien passé de la représentation à la modélisation qui, en tant que recomposition du monde, s'imposerait comme acte quasi démiurgique? La question mérite d'être posée car si, historiquement, l'art se définissait surtout en tant que production d'une œuvre créée à partir d'un modèle concret, emprunté au réel dont elle serait la représentation, force est de constater que 
cette définition aujourd'hui n'opère plus, du moins lorsqu'on aborde tout un pan du théâtre contemporain, et plus particulièrement le théâtre de l'image. La scène ne lève plus le rideau sur une "tranche de vie». Actuellement, les créateurs semblent retrouver le mouvement des choses et en rendre compte, de même que l'acte de création semble s'ancrer dans le principe du mouvement qui anime et qui métamorphose, principe qui meut l'œuvre vivante. Le théâtre de l'image, procédant à un immense brassage d'éléments hétérogènes, en les déconstruisant et en les reconstruisant, dévoilerait les pièces et le fonctionnement de ce mécanisme obscur qu'on appelle «création». C'est ainsi qu'une œuvre prend vie et corps sous les yeux du spectateur, lui procurant le sentiment de capter le moment fugitif de sa création ou d'y assister. Le spectacle que nous offre le théâtre actuel serait-il donc celui de l'accouchement artistique considéré comme un processus vivant, comme une épigénèse riche en surprises, en métaphores et en métamorphoses ${ }^{9}$ ?

\section{VINCI: UN VOYAGE INITIATIQUE}

Ne pouvant aborder tout le théâtre de l'image, nous tirerons nos exemples de Vinci ${ }^{10}$, œuvre emblématique de celui qui, au Québec, a la réputation de véritable "faiseur d'images", l'auteur, metteur en scène, cinéaste et comédien Robert Lepage. L'œuvre de cet artiste prolifique et polyvalent est non seulement représentative de la nouvelle poétique qui semble sourdre de l'ère informatique mais constitue un terrain de choix permettant de mieux rendre compte des conséquences épistémologiques du croisement des médias dans la production culturelle contemporaine et de la manière dont le théâtre de l'image participe au renouveau de l'écriture et de la pensée. À un moment où s'implante un nouvel ordre visuel - qui est en train de rompre avec les règles de représentation implantées depuis la Renaissance (et de ce point de vue la référence à Léonard de Vinci ne serait pas fortuite) -, ce que son théâtre explore au moyen de l'image et au-delà de celle-ci, ce sont précisément les codes du visible tels que bouleversés par l'impact de la technologie. Si, comme dit Lepage, «les médias ont changé et changent notre façon de penser, autant sur le plan de la forme que du contenu " 11 , ce que l'image théâtrale en tant que médium est en train de changer, c'est particulièrement la façon séculaire de voir, en exposant sur la scène - c'est-à-dire en mettant sous les yeux - le nouveau regard que notre époque pose sur le monde.

La spécificité du théâtre de Lepage repose sur une écriture scénique qui procède par collage, montage, assemblage ou bricolage de sons, de lumières, d'objets, d'appareils technologiques, d'écrans, etc., bref, d'autant d'éléments disparates et hétérogènes qui s'offrent comme ressources sensibles potentiellement exploitables tout au long du processus de création. En cédant ainsi l'initiative aux éléments de la scène plutôt qu'à un texte préétabli, ce théâtre visuel met en évidence deux principes qui se recoupent: le premier pointe l'une des assises du fonctionnement de l'image, soit la nature spatiale de sa structure qui est assemblage, combinaison; le second épingle le fondement de la pensée qui est montage. C'est ce que nous révèle l'étude de Vinci, spectacle conçu, réalisé et interprété par Robert Lepage qui occupe seul la scène pendant un heure et demie et qui, glissant du XXe siècle à la Renaissance, propose une réflexion sur l'art, segmentée en un itinéraire d'une dizaine de tableaux:

Chaque tableau correspond à une étape du voyage initiatique de

Philippe, un jeune photographe québécois à la recherche de sa

vérité artistique: parvenir à créer de façon intègre dans un monde où les compromis sont nécessité et loi. 12

Nous résumons brièvement. C'est la mort de Marc, un ami cinéaste, qui s'est suicidé plutôt que d'être infidèle à son art, qui fait surgir en Philippe de nombreuses questions sur lui-même, sur l'art et la création. À la suite de quelques séances de psychanalyse, Philippe décide de s'envoler pour l'Europe.
La trajectoire du personnage va de Québec à Vinci, en passant par Londres, Paris, Florence et par la rencontre et la confrontation avec différents guides (psychologiques, touristiques, artistiques), et surtout, à travers eux, avec lui-même. 13 
Ce voyage sera pour Philippe l'occasion de vaincre son angoisse principale: la peur du vide, de l'abandon dans la création artistique.

\section{A STRANGE IMPRESSION OF... DÉCALAGE}

Vinci commence avec l'arrivée d'un guide aveugle portant des lunettes noires qui, sur une musique orientalisante, s'adresse en italien au public, agissant un peu à titre de présentateur. Sur scène, cette présentation est faite devant un écran (qui avec quelques panneaux vitrés constituent le seul décor de Vinci), écran sur lequel est projetée une traduction française du discours du guide qui se tient debout devant un train électrique en mouvement. Ce train servira de métaphore aux considérations sur l'art qui traversent toute la pièce: «l'arte è un veicolo». Philippe, le jeune photographe qui, aux dires de ses amis, se pose trop de questions sur l'art, arrive d'abord à Londres et subit son premier choc (tableau 2: Big Ben, London). Un guide touristique britannique s'adresse, sur un ton particulièrement condescendant, à un groupe de «little French Canadians» montés à bord d'un autobus pour visiter la ville. L'intérieur de l'autobus, le rétroviseur et le guide - dont la figuration tient dans la seule silhouette de la casquette - sont vus en ombres chinoises. Les Londoniens conduisent à gauche, explique-t-il, ce qui procure «a strange impression of... décalage». La solution? «Look into to mirror... or open the window", conseille le guide. Philippe découvrira progressivement que la réalité peut arborer plusieurs masques. Le spectateur découvrira à son tour que Robert Lepage joue avec art $\mathrm{du}$ contrepoint en superposant les motifs figuratifs formels, incidemment théâtre et ombres chinoises (ce qu'il fera à plusieurs reprises dans le spectacle), installant une sorte de relation dialogique entre la projection en aplat (propre à la peinture, au cinéma et à la vidéo) et le jeu en trois dimensions (propre au théâtre), démarche artistique contribuant à interroger la forme «image» - en surface et en profondeur, statique et animée, réelle et virtuelle - par laquelle passe tout le réel perçu. Ne peut-on pas penser que dans l'observation du monde physique qui est pointée ici, de façon triviale peut-être (les Londoniens conduisent à gauche, ce qui procure une «impression de décalage»), vient en quelque sorte se réfracter l'idée selon laquelle, dans la figuration, tout est affaire de perception. Aussi, quel que soit le traitement auquel le «visible» sera soumis pour répondre aux lois de la représentation, il nous faudra toujours des outils pour nous aider à surmonter nos problèmes de lisibilité, de décalage, et ce, que nous soyons créateur ou spectateur. Si le miroir était, pour les peintres de la Renaissance, un de ces instruments privilégiés permettant de corriger les imperfections de la vue, ce n'est peut-être plus l'objet le plus adéquat aujourd'hui pour venir soutenir notre perception de la réalité sensible... en dépit de ce qu'en dit le guide britannique dans la pièce. Les «imperfections de la vue», qui ont préoccupé les artistes à travers l'histoire de l'art, pourraient peut-être moins tenir de l'élaboration d'une science et d'un art du visible soumis à la représentation et à ses règles de composition (celles mises en place depuis la Renaissance, comme nous l'avons dit) que de la vision même que nous avons des choses, autrement dit du fait que toute figuration (peinture, dessin, photo, cinéma, théâtre) est image d'image et qu'en tant que telle, elle ne sera jamais copie conforme de la «réalité sensible» ou du «visible».

Nous sommes tentées de dire tout de suite qu'avec le théâtre de l'image, on passe de l'ordre de l'écriture, qui s'ordonne en fonction des mots que l'on aligne l'un après l'autre, à l'ordre de la vision qui fait plutôt appel à la contiguïté et qui, de ce fait, risque davantage la contamination. Autrement dit, on passe de l'homogénéité de la parole écrite à l'hétérogénéité de la vision. Car, par opposition à l'écriture qui présuppose ordre, raisonnement et savoir, la vue, qui est polydirectionnelle, parait trouble, anarchique. Aussi, pour voir, il faut découper; mais il faut avant tout percevoir. Et dès qu'on perçoit, on est en acte de cognition et, par conséquent, en acte d'interprétation, voire d'auto-interprétation, c'est-à-dire que l'on fragmente, sélectionne, réorganise en "créant", à son tour et à l'instar des acteurs, des configurations plus 
ou moins originales de ce que l'on voit. L'idée avancée ici d'auto-interprétation, comme véritable plan focal du théâtre de l'image, déborde donc largement le simple fait de reconnaissance des images du monde, de sorte que la notion de signification, chère à la mise en scène traditionnelle, ne peut plus être posée $a$ priori, c'est-à-dire comme sens intégral et autonome, alors que le logocentrisme paraît ébranlé dans ses fondements.

\section{L'ARTE È UN VEICOLO}

Quittant Londres, le photographe (Philippe) arrive à Paris et rencontre la Mona Lisa dans un Burger King du boulevard Saint-Germain (tableau 4: Burger King, boulevard Saint-Germain, Paris 5e). Que penser de cet échange "interculturel», de cette surimpression baroque de temps et d'esthétiques? Pure fantaisie postmoderne? Ou structure intertextuelle accordée à l'esprit du temps, qui révèle le besoin fondamental de bricolage dans toute activité créatrice, fissurant le mythe du texte clos. Il est clair que le travail de composition du théâtre de l'image relève du bricolage 14 en raison notamment de ses jeux d'associations, comme celui que nous venons d'évoquer ( «travestissement» d'un modèle italien du XVe siècle en Parisienne «branchée» de 1985), mais il appert aussi que celui-ci offre au-delà de ses représentations inédites, fondées bien souvent sur des agencements composites, des images complexes conçues comme métaphores de la réalité perçue. Or les stratégies métaphoriques qui animent ce «bricolage» patent du théâtre de l'image constitueraient «une boucle constructive [...] qui part de l'œil [...] pour retourner à l'œil [...] en re-construisant un monde à partir des “échantillons prélevés” " 15 . C'est en fouillant la mémoire et en prélevant donc des échantillons à d'autres disciplines artistiques (peinture ${ }^{16}$, cinéma ${ }^{17}$, littérature ${ }^{18}$ ) et à d'autres cultures (orientale ${ }^{19}$, européenne et américaine ${ }^{20}$ ) que le théâtre de l'image ouvrirait sur l'universel, expérimenterait et révélerait un nouveau rapport au cosmos. Ceci parce que le mixage des références et des fragments (on pourrait dire aussi la construction par épisodes ou par «attractions» pour reprendre le langage d'Eisenstein), dont Vinci est emblématique, opère chaque fois comme une plongée dans l'espace sensoriel et mnésique individuel - donc subjectif -, empruntant le chemin, non pas de la démonstration ou de la connaissance logique, linéaire, déterministe, mais plutôt celui de l'hypothèse analogique ou de la métaphore qui, sous une allure de baguette magique, mène à la modélisation. Par ces références ou emprunts aux autres arts et aux autres cultures, perçus parfois comme des dérapages ou des décalages, le théâtre de l'image en arriverait à faire glisser son spectateur vers un nouvel espace perceptif, celui de la conscience qui conçoit, qui interprète, celui de la fabrication intérieure de l'image, ouvrant par ce mouvement, par ces déplacements, par ce jeu, de nouveaux territoires à la connaissance et à la pensée, faisant finalement et par analogie la démonstration du leitmotiv de Vinci: «l'arte è un veicolo».

Au lieu donc d'imiter les apparences, le théâtre de l'image impose au regard des décalages visuels qui, ébranlant les modalités de la perception, remettent en question la notion traditionnelle de représentation en tant que copie plus ou moins conforme à un réel conçu pendant longtemps comme stable et objectif. La représentation désormais (tant la représentation théâtrale que la représentation mentale) est perçue - et, par conséquent, devrait être analysée - comme étant travaillée, œuvrée par la mémoire, l'imaginaire et le monde, passant du donné vers le construit, amorçant un nouvel ordre artistique, une nouvelle poétique capable de remettre en question les modalités de la perception parce que sachant déverrouiller la réalité. Ne serait-ce pas le propos ou en tout cas un des sens possibles de la séquence suivante?

\section{BRICOLAGES}

Nouvelle métamorphose de Lepage -qui interprète à lui seul tous les personnages de la pièce -en Mona Lisa (tableau 5: Mona Lisa, huile $77 \times 53 \mathrm{~cm}$, le Louvre, Paris). La Joconde démythifiée, personnage pictural en deux dimensions, a ici vie et épaisseur. Elle raconte que 
le paysage dans lequel «un ami» l'a peinte autrefois est celui qui succède à une catastrophe atomique:

Mes mains, dit-elle, étaient sur mon ventre, comme si je portais en moi l'embryon d'une génération nouvelle d'enfants-lumière. Et pour bien faire comprendre que j'étais la seule à savoir, il m'avait peint un sourire énigmatique. (Extrait de Vinci)

Ébranlant le savoir, la figure que le créateur tente de restituer ici ne serait-elle pas celle du paradoxe? Car dans le «morceau de réalité» prélevé, Robert Lepage ne fait pas que reproduire passivement l'héritage de la culture. En "grand imagier " ${ }^{21}$ qu'il est, il incarne d'abord le plus «réalistement» et mimétiquement la figure à représenter, incidemment la Joconde, pour ensuite s'affranchir librement de la sujétion au modèle emprunté au réel, effaçant ainsi tout résidu naturaliste.

Admettant, comme nous l'avons fait, que l'image n'est pas juste une saisie, mais un acte originel d'organisation, on comprendra que le créateur ne veut pas se limiter à redoubler le monde; s'il le fait, c'est pour mieux s'en écarter, c'est pour mieux en jouer. Or ce jeu tient pour beaucoup dans le nouveau regard qu'il porte, autrement dit dans la pensée créatrice qu'Edgar Morin définit comme suit en reprenant les mots de Szent-Gyorgÿ:

[la pensée créatrice, la découverte] consiste à voir ce que tout le monde a vu et à penser ce que personne n'a pensé. [...] L'imagination élabore des formes ou figures nouvelles, elle invente/crée des systèmes à partir des éléments captés ici ou là ou détournés des systèmes dont ils faisaient partie, ce qui confirme, dans la sphère de la pensée, le caractère bricoleur de toute évolution créatrice. 22

Robert Lepage ne manifeste-t-il pas son aptitude à bricoler en détournant la Joconde de son système premier de référence pour l'intégrer dans un nouveau système? Quant à la transformation opérée, elle résulterait de l'assemblage d'éléments, opération qu'il est tentant de rapprocher du montage cinématographique, comme activité structurante. André Gaudreault ne dit-il pas que «c'est par le montage que le narrateur peut se permettre d'exercer son pouvoir sur le narré». «C'est ni plus ni moins par les raccords qu'il parle» 23 . Passons donc au prochain raccord.

\section{OPEN THE WINDOW...}

Il s'agit de la séquence où le guide italien, aux lunettes noires, imprime un mouvement très rapide à sa canne blanche avant de conclure que la différence entre l'art et la mort est simplement une question de vitesse. Sur scène, ce geste de la main qui agitait à grande vitesse la canne créait une sorte d'écran immatériel sur lequel étaient projetées des diapositives d'œuvres d'art. "Ces visions furtives détachées sur l'obscurité et constituées de pur mouvement et de pure lumière [semblaient] établi[r] la marge très mince qui existe entre ouvre et néant» 24 . Or ne se pourrait-il pas que, comme le soutient Pierre Ouellet,

[...] les images so[ie]nt moins des écrans, où se re-présenterait le monde projeté [...] que de véritables fenêtres [...] où le monde se dé-présente dans les limites, réelles mais irréalisantes, de l'ouverture que l'imagination poiétique pratique dans le mur aveugle de la spatialité pure, telle une déchirure par où la pensée passe[rait] avec le regard. 25

Auquel cas, les «fenêtres fictives» ainsi ouvertes («Open the window", conseillait le guide britannique; $c f$. A strange impression of... décalage) seraient «du même coup défenestration: [i.e.] précipitation, par cette déchirure même [que l'œuvre d'art] ouvre dans l'espace, d'où le regard et tout le corps avec, âme comprise, se jettent sur les "choses" du monde»" ${ }^{26}$. N'est-ce pas ce que nous suggère la dernière séquence de Vinci?

\section{MÉTAPHORES VISUELLES}

Au terme de son voyage, Philippe déployant d'immenses ailes, semblables à celles qu'avait imaginées et dessinées Léonard de Vinci, vainc sa peur du vide et réalise un envol triomphal (tableau 9: Vinci, un mont planté d'oliviers). C'est, en effet, du haut de la falaise surplombant le village de Léonard que Philippe ose s'abandonner et plonger dans l'infini, ayant enfin saisi le secret de la création artistique que le guide florentin avait un peu plus tôt tenté de communiquer: «l'imagination, avait-il dit alors, procure un envol 
libérateur». Cette phrase, il l'avait prononcée alors qu'il faisait visiter à un groupe de photographes la Piazza del Duomo de Florence (tableau 7: Duomo, Firenze). Seul, sans accessoires, il les avait promenés du baptistère au campanile et à l'ensemble de la cathédrale, ne parcourant la scène qu'en largeur, et disant ne pas voir les richesses qu'il décrivait:

S'il est parmi vous des gens qui, comme moi, ne peuvent pas voir tous ces vitraux, ces coupoles, ces chefs-d'œuvre, que quelqu'un vous les suggère simplement et que vous les voyez de l'intérieur; vous êtes à ce moment-là un aussi grand architecte que Léonard de Vinci, car l'imagination procure un envol libérateur... 27

Il est significatif que ce soit le guide aveugle qui comprenne

[...] la uraie beauté des choses et a de cette beauté une vision intérieure qu'il tente de nous transmettre. [...] Possédant les yeux de l'âme et de l'imagination [...] il appara[ît] [...] comme l'image même de la liberté créatrice. 28

À elle seule cette séquence donne à penser que le spectateur doit, à la suite du créateur, accepter de jeter son regard dans la déchirure du mur du monde et forcer, à l'instar du guide italien, la mémoire et l'imagination à faire entrer l'«impossible» dans l'«apparence réelle» et, de ce fait, arriver à surmonter les problèmes de lisibilité. Si bon nombre de textes dramatiques et spectaculaires contemporains présentent certaines obscurités, celles-ci tiendraient peut-être du fait - et c'est ce que viendrait nous rappeler le théâtre de l'image par la voie de la métaphore - que la création, même à l'heure du visuel - et peut-être plus à l'heure du visuel -, demeure une activité de l'intérieur. Il devient de plus en plus évident que percevoir n'a rien de passif.

Une sorte d'équivalence peut ici être établie entre le travail de "lecture» et celui de «l'écriture» ou entre le travail non pas tant de "réception» que de "perception" et celui de "création", venant nous rappeler le rôle de premier plan qui est dévolu au spectateur ${ }^{29}$. Il est intéressant de signaler au passage que la conception qu'avait Eisenstein du cinéma au début du siècle tenait déjà de cette approche, à savoir que «[...] le spectateur est entrainé dans un acte de création au cours duquel sa personnalité, loin d'être asservie à celle de l'auteur, s'épanouit [...]»30. Et pour mieux comprendre ce qui peut déterminer (asservir ou émanciper) sa vision, il faut, comme Philippe, vaincre sa peur et se jeter dans le vide; car, comme l'explique encore Pierre Ouellet,

[l'] eil n'est pas un organe réceptif, qui se contente de capter les stimuli de son environnement; organe préhensif, il va chercher, chasser même [...] au sein du monde dans lequel il baigne les aspects qui l'attirent [...] Le regard va à ce qu'il voit, se projette dans l'espace, s'y fraye un chemin, parcourt l'étendue, explore ses recoins [... au point de nous faire] associer vision et motion en une même expérience esthétique. 31

Or cette association de la vision et de la motion n'est-elle pas au cœur du cinéma et du théâtre? Mais à la différence du premier qui, au début du siècle, s'est présenté comme un art du mouvement, le théâtre de l'image s'impose, à la fin de ce même siècle, comme un art en mouvement. Un art qui s'intéresse à toutes les nuances du changement, de l'imitation à la métaphore, et qui cherche moins à faire voir le mouvement du monde réel qu'à poser le regard sur lui-même (pour ne pas dire sur la pensée) et, incidemment, sur ses propres mouvements et mécanismes obscurs. Car le théâtre de l'image semble avoir inventé une trajectoire fonctionnant surtout par captation métaphorique, c'est-à-dire par transport ou déplacement non pas du monde réel ou de son image, mais bien du regard tant de l'artiste que du spectateur. Si cette trajectoire métaphorique se concrétise, sur le plan du contenu, par la thématique du voyage (omniprésente chez Lepage), elle s'avère, sur le plan formel, une véritable métaphore visuelle, sorte d'accélérateur de la vision qui déforme ou, mieux, transforme l'aspect habituel des choses, les donnant à voir sous d'autres angles, sous d'autres aspects et, bien souvent, sous celui du «jamais-vu». La métaphore visuelle s'érige ainsi en figure centrale de l'écriture scénique, machine qui «motive l'artiste», dispositif qui le «locomotive», comme l'explique le présentateur italien dans Vinci ${ }^{32}$, tout en stimulant la 
«locomotion» du regard, de l'imagination, de la mémoire, bref, de la pensée du spectateur.

Il semble bien que ce que raconte ou donne à voir Vinci, c'est qu'au-delà de tous les appareils qu'on a mis au point depuis la Renaissance pour réfléchir la réalité sensible, le dispositif le plus raffiné sera toujours celui qui sera capable de générer des images en imagination, de mettre l'imagination elle-même en mouvement. Se pourrait-il qu'en dépit de la poussée des technologies nouvelles, qui ont contribué à l'essor de la culture médiatique, la motricité recherchée soit désormais moins celle de l'image (puisque celle-ci est acquise depuis l'avènement du cinéma et des images cinétiques) que celle de la pensée? N'est-ce pas là le sens du message livré par le personnage du guide aveugle dans Vinci, qui finalement voyait avec les yeux de l'âme et de l'imagination? Pourrions-nous voir à notre tour, dans ce tableau (tableau 7: Duomo, Firenze), la métaphore de la remise en cause du dualisme fondateur de notre espace de représentation classique? Et pourrions-nous penser, du coup, que la coupure entre vu et voyant autour de laquelle s'articulait la vieille relation spectaculaire, illustrée par la rampe séparant la scène de la salle, serait maintenant suturée? Le dispositif commun au théâtre et au cinéma, opposant une salle obscure à une révélation lumineuse, viendrait-il d'être brisé, sinon du moins ébranlé? Et l'image pourrait-elle enfin être voie d'accès à l'immatériel, à l'invisible, ainsi que l'a toujours envisagé l'art oriental?

Pour l'heure, on peut penser que le théâtre de l'image, et plus particulièrement le théâtre de Robert Lepage qui nous intéresse ici, nous mettrait en présence d'une nouvelle forme d'écriture-langage, dynamique et interactive, que l'on pourrait qualifier, selon l'expression de Pierre Lévy, de "pensée-image» 33. Loin d'être simple illustration animée de la pensée, la "pensée-image» serait instrument de connaissance et de pensée, tout en étant image animée. Chose certaine, l'écriture scénique de Lepage se présente comme une écriture dynamique, dont les symboles sont porteurs de mémoire tout en étant dotés d'une capacité de réaction autonome. Ce qu'il est important de comprendre c'est que les caractères de cette écriture ne signifient pas seulement par leur forme ou leur disposition, mais surtout par leurs mouvements et leurs métamorphoses. Aussi ce théâtre offre-t-il des images insolites, des modèles inédits qui, bouleversant les habitudes perceptives du spectateur, mettent en doute tant sa faculté de voir que sa logique traditionnelle.

\section{UN ART EN MOUVEMENT}

Pour juger de l'importance de la transformation opérée par le théâtre de l'image et plus particulièrement par l'écriture dynamique ou «penséeimage» de Lepage, il importe de se rappeler que l'écriture, depuis l'origine, s'est toujours déployée sur un support statique, utilisant des symboles fixes (alphabets ou idéogrammes) qui se succèdent linéairement. Si, avec l'avènement du cinéma et de la télévision, le mouvement et l'image ont pris le pas sur le langage linéaire et statique, générant un langage bidimensionnel et animé, ce dernier toutefois n'a pas la propriété d'être interactif. Or c'est précisément cette propriété que va réussir à installer le théâtre de l'image en usant d'un langage qui n'est pas construit $a$ priori, mais se présente plutôt comme une sorte de répertoire d'idéogrammes en évolution constante, et dont la saisie du sens n'est possible que dans la mesure où le spectateur s'y investit.

Élaborée de façon interactive, cette nouvelle écriture scénique est bien plus qu'une simple question de mode. En y regardant de près, nous constatons qu'elle s'accorde de façon étonnante avec cette écriture dynamique à base d'icônes, de schémas et de réseaux sémantiques, inventée par les nouvelles technologies de communication qui, soutient Lévy, stucturent déjà «une part significative de la création artistique contemporaine avant même son informatisation" 34 . Cette "concordance» formelle serait donc plus qu'une simple coïncidence ${ }^{35}$. Dans cette perspective, les juxtapositions audacieuses de références à d'autres cultures et à d'autres disciplines ainsi que l'utilisation de nouvelles technologies, pratiquées par le théâtre de l'image, seraient plus logiques qu'elles en ont l'air, et surtout rappelleraient 
que le théâtre est mémoire, en même temps qu'acte indispensable d'adaptation au réel. Alors donc qu'il semble s'affirmer comme référent métaphorique de l'architecture cognitive individuelle et collective, le théâtre de l'image ne participerait-il pas, à sa manière, au renouveau contemporain de l'écriture et de la pensée? Et, avec lui, ne serait-il pas en train de se dessiner une nouvelle poétique adossée à l'imaginaire contemporain et à l'environnement dans lequel nous vivons? Une sorte de poétique du jeu libre et fluide, de la construction par fragments ou par attractions, mais surtout de la construction en mouvement, ouvrant, par ses plongées dans le vide (cf. Open the window) et par ce qui vient frapper le regard, de nouveaux territoires à la création, à la connaissance et à l'expression visuelle de la pensée. Alors que le théâtre et le cinéma peuvent aujourd'hui jouer à loisir de la stylisation ou de stratégies illusionnistes, maîtrisant les modes de représentation du volume, de la profondeur et de l'animation, la métaphore visuelle dont use le théâtre de l'image serait-elle à présent le principe clé d'une conception neuve, même si elle constitue un terrain qu'il faut croire encore traumatisant pour la production visuelle? Il nous faut conclure, et nous laisserons cette question ouverte pour en poser quelques autres.

Si nous pouvions résumer visuellement la Renaissance avec un Léonard, et qu'on nous demandait de faire le même exercice pour résumer la trame mentale et la sensorialité du XXe siècle, quelle(s) œuvre(s) choisirions-nous? Nous aurions probablement avantage à afficher - au minimum - un programme double. La première partie pourrait s'ouvrir par une projection des frères Lumière mais, si nous voulons témoigner de l'ère nouvelle dans laquelle, selon Régis Debray, nous sommes maintenant entrés, celle dite du visuel, comme il l'appelle, quelle ouvre retiendrionsnous? «Nous étions jusqu'à présent devant l'image, écrit-il, nous sommes désormais dans le visuel " ${ }^{36}$. Ne serait-ce pas un des sens de l'allégorie visible du regard qu'a porté Lepage sur Vinci en lui donnant une autre vie? Que faire voir donc, en deuxième partie, comme image capitale d'une génération dont on ne cesse d'exciter l'imagination ludique? Le théâtre de Lepage? Mettons. Mais en raison de l'éphémérité même du théâtre qui ne laisse pas de traces, on devra alors chercher à faire image pour que s'active l'imaginaire et se mette en branle la perception. En d'autres mots, mettre en marche «l'atelier de la pensée», comme le disait Shakespeare. Instituer un mode de communication où le distinguo narration/monstration, tel qu'opéré par André Gaudreault pour marquer les points d'ancrage du système filmique, consiste ici à rapporter, à dire ce qu'ont fait certains personnages dans une pièce, pour montrer non pas tant leurs agissements (comme le préconisait Aristote dans sa Poétique), que d'abord et surtout le travail, le mouvement, les actions de la pensée à laquelle doit être insufflé un dynamisme tel qu'elle puisse prendre son envol et mener le spectateur à la jonction ou aux confins des mondes visible et invisible.

Il est clair qu'ayant abattu la barrière des codes de l'illusionnisme et du réalisme qui ont longtemps maintenu le regard occidental dans une sorte de "naïveté», le spectateur actuel ne sera jamais plus effrayé à la vue de l'arrivée en gare du train de Lumière; il pourra cependant craindre de plonger dans le vide de l'espace de l'imaginaire dans lequel cherche à le faire basculer le théâtre de l'image, ne sachant trop où cela peut le mener, à quelle révélation le saut peut le conduire, maintenant qu'est loin derrière lui l'espace unitaire de la Renaissance. C'est pourtant l'exigence à laquelle il est confronté, invité qu'il est à présent à réfléchir sur l'imaginaire de la réalité et la réalité de l'imaginaire, comme le suggère Edgar Morin. Ce serait donc au spectateur qu'incomberait de faire tenir ensemble les fragments de l'œuvre comme autant «d'échantillons du monde» prélevés, et de combler par l'imagination les trous laissés par les pièces manquantes du puzzle. Alors que la scène théâtrale semble être devenue à présent le milieu ou le creuset d'un «in-lusio» - au sens d'entrer dans le jeu - intermédiatique, ne serait-ce pas à nous spectateurs de faire la preuve de la faculté combinatoire qu'a notre propre imagination de faire tenir ensemble une série d'images... comme le fait, à sa façon, le cinéma et, à la sienne, le théâtre? Et 
pour ce faire, ne disposons-nous pas de la "machine" la plus fabuleuse, notre cerveau, notre pensée perceptive, notre pensée créatrice? De ce défi à relever, semble dépendre notre condition d'aveugle ou de voyant. Saurons-nous devenir cette génération d'enfants-lumière (cf. Bricolages) dont la Joconde de Robert Lepage disait porter en elle l'embryon?

\section{N O TES}

1. J.P. Ryngaert, 1993: 5.

2. Pour en savoir davantage sur cette mutation qu'a connue la pratique théâtrale québécoise des vingt dernières années, on pourra consulter: C. Hébert et I. Perelli-Contos, 1994a : 64-73; 1993: 9-13.

3. V. Meyerhold, $1980: 217$.

4. Ibid., p. 219

5. Ibid.

6. G. Lista, 1989: 256 .

7. Ibid.

8. Ibid., p. 90.

9. C'est en tout cas ce que nous donnent à penser bon nombre de spectacles de Robert Lepage. Si nous nous attardons ici à Vinci, on trouvera ailleurs des analyses d'autres de ses spectacles. Par exemple, qui serait intéressé au spectacle Les Aiguilles et l'Opium pourra lire le texte de C. Hébert, 1997 : 25-39.

10. Vinci, spectacle conçu, réalisé et interprété par R. Lepage, a été créé en 1986, au Théâtre de Quat'Sous à Montréal. Qui voudrait lire une autre étude de Vinci pourra également consulter C. Hébert, 1994: 54-58. 11. Propos recueillis par C. Hébert et I. Perelli-Contos, 1994b : 64.

12. J. Bovet, 1990: 95.

13. Ibid.

14. Voir à ce sujet l'article d'I. Perelli-Contos, 1988: 322, ou encore celui de C. Hébert et I. Perelli-Contos, 1993: 9.

15. E. Morin, 1986: 108

16. Comme dans Vinci (Robert Lepage).

17. Comme dans Le Bord extrême (spectacle mis en scène par M. Nadeau et R. Lepage, 1986) et Le Polygraphe (spectacle conçu par R. Lepage et M. Brassard, 1988).

18. Comme dans Les Aiguilles et l'Opium (spectacle conçu et mis en scène par R. Lepage, 1991) et dans Elseneur (spectacle conçu et interprété par R. Lepage, 1995).

19. Comme dans En attendant (spectacle mis en scène et interprété par R. Fréchette, J. Lessard et R. Lepage, 1982), dans La Ttrilogie des dragons (spectacle de et avec M. Brassard, J. Casault, M. Gignac et R. Lepage, 1985) et dans Les Sept Branches de la rivière Ota (spectacle mis en scène par R. Lepage, 1995).

20. Comme dans Les Aiguilles et l'Opium (R. Lepage).

21. L'expression est d'A. Gaudreault, 1988: 107.

22. E. Morin, 1986: 188 et 189 .

23. A. Gaudreault, 1988: 112 et 114.

24. D. Pavlovic, $1987: 97$

25. P. Ouellet, 1995: 15.

26. Ibid., p. 16.

27. Extrait du scénario Vinci de R. Lepage (Productions Téléféric, tapuscrit non paginé, s.d.).

28. D. Pavlovic, 1987: 99.

29. Sur le nouveau rôle qu'est appelé à jouer le spectateur, on pourra consulter I. Perelli-Contos, 1994: 203-211; 1997.

30. S. Eisenstein, 1958: 93.
31. P. Ouellet, 1995: 12.

32. Au premier tableau, le guide aveugle, seul debout, au centre de la scène, explique les enjeux du spectacle: «[...] le train qui tourne depuis le début est une façon visuelle de vous communiquer que L'ART EST UN VÉHICULE». Il fait allusion au train électrique en mouvement non loin de lui (cf. A strange impression of... décalage).

33. P. Lévy, 1991: 11.

34. Id., $1987: 89$.

35. Pour en savoir davantage sur l'influence technologique exercée sur tout un pan du théâtre actuel et sur nos modes de pensée, on pourra consulter C. Hébert et I. Perelli-Contos, 1998: 171 à 204. On pourra lire aussi la thèse de doctorat de C. Loffree, 1999.

36. R. Debray, 1992 : 298

\section{RÉFÉREN CES BIBLIO GRAPHIQ UES}

BOVET, J. [1990] : «Le symbolisme de la parole dans Vinci », L'Annuaire Théâtral, no 8 , Montréal, Société québécoise d'étude théâtrale. DEBRAY, R. [1992]: Vie et mort de l'image. Une histoire du regard en Occident, Paris, Gallimard.

EISENSTEIN, S. [1958]: Réflexions d'un cinéaste, texte russe établi par R. Iourénev, trad. par L. et J. Cathala, Moscou, Éd. du Progrès.

Gaudreault, A. [1988]: Du littéraire au filmique, Québec/Paris, Presses de l’Université Laval/Méridiens Klincksieck.

HÉBERT, C. [1997]: « De la mimesis à la mixis ou les jeux analogiques du théâtre actuel", dans C. Hébert et I. Perelli-Contos, Théâtre, mulidisciplinarité et multiculturalisme;

[1994]: «L'écriture scénique actuelle: l'exemple de Vinci », Nuit blanche, $\mathrm{n}^{\circ} 55$, mars, avril, mai, 54-58.

HÉBERT, C. et I. PERELLI-CONTOS [1998] : «L'écran de la pensée ou les écrans dans le théâtre de Robert Lepage», dans Les Écrans sur la scène.

Tentations et résistances de la scène face aux images, sous la dir. de B. Picon-

Vallin, Lausanne, L'Âge d'Homme, 171-205;

[1997]: Théâtre, mulidisciplinarité et multiculturalisme, Québec, Nuit blanche éditeur

[1994a]: «Une mutation en cours", Théâtre/Public, n 117, Théâtre de Gennevilliers, mai-juin, 64-73.

[1994b] : «La tempête Robert Lepage », Nuit blanche, no 25, mars, avril, mai ;

[1994c] : «Le théâtre avec ou sans texte », dossier préparé pour la revue Nuit blanche, no 55 , mars, avril, mai, 44-71;

[1993] : «Les lointains de la pensée comme enjeux du théâtre de

recherche ", Éducation et francophonie, Québec, Association canadienne

d'éducation de langue francaise (ACELF), vol.XXI, nº 2, 9-13.

LÉVY, P. [1991] : L'Idéographie dynamique, Paris, La Découverte;

[1987]: La Machine univers, Paris, La Découverte.

LisTA, G. [1988]: La Scène futuriste, Paris, Éd. du Centre National de la

Recherche Scientifique, 1989.

LOFFREE, C. [1999]: Le Théâtre québécois contemporain à la lumière de la culture informatique, Sainte-Foy, Université Laval.

MEYERHOLD, V. [1980] : Écrits sur le Théâtre, 1930-1936, tome III, Lausanne, La Cité-L'âge d'homme.

MORIN, E. [1986]: La Méthode 3. La Connaissance de la Connaissance, Paris, Seuil.

OUELLET, P. [1995]: «L'image mue; vision et motion dans le langage poétique", Protée, vol. 23, nº 1 , hiver.

PAvlovic, D. [1987] : «Du décollage à l'envol», Cahiers de théâtre Jeu, no 42

(dossier spécial sur Vinci), Montréal, les Cahiers de théâtre Jeu.

Perelli-Contos, I. [1997] : «De "l'art” du spectateur », dans C. Hébert et I.

Perelli-Contos, Théâtre, multidisciplinarité et multiculturalisme;

[1994]: «L'acte de lecture au théâtre», dans L'Acte de lecture, sous la dir. de

D. Saint-Jacques, Québec, Nuit blanche éditeur;

[1988] : «Le discours de l'orange », L’Annuaire Théâtral, nos 5-6, Montréal, Société québécoise de recherche théâtrale, automne et printemps. RYNGAERT, J.-P. [1993] : Lire le théâtre contemporain, Paris, Dunod. 